\title{
Mycobacterium fortuitum-related lymphadenitis associated with the varicella-zoster virus
}

\author{
Kleber Giovanni Luz ${ }^{[1]}$, Maria Helena Marques Fonseca de Britto ${ }^{[2]}$, \\ Domitila Costa de Farias ${ }^{[3]}$, Mayara Varaschim Almeida ${ }^{[3]}$, \\ Nastassja Morgana de Sousa Figueirêdo ${ }^{[3]}$ and Paula de Medeiros Nacácio e Silva ${ }^{[3]}$
}

[1]. Departamento de Infectologia, Universidade Federal do Rio Grande do Norte, Natal, RN. [2]. Centro de Patologia Clínica, Natal, RN. [3]. Curso de Medicina, Universidade Federal do Rio Grande do Norte, Natal, RN.

\begin{abstract}
Lymphadenitis caused by non-tuberculous mycobacteria is an uncommon manifestation in immunocompetent individuals. Here, we report a case of Mycobacterium fortuitum infection in a previously healthy 9-year-old patient who developed cervical lymphadenitis evolving to a suppurative ulcer associated with a varicella-zoster virus infection. We discuss the relationship between the varicella-zoster virus and the immune response of the host as an explanation for the unusual progression of the case.
\end{abstract}

Keywords: Lymphadenitis. Non-tuberculous mycobacteria. Varicella-zoster virus. Mycobacterium fortuitum.

\section{INTRODUCTION}

Non-tuberculous mycobacteria (NTM) are ubiquitous organisms found in soil and water ${ }^{1,2}$. They are of variable pathogenicity and are able to colonize a host and influence the occurrence of infection or disease. NTM require an opportunistic opening ${ }^{2}$ to inoculate an individual and will usually take advantage of skin or mucosal barrier breaches. NTM primarily cause pulmonary, skin, and soft tissues diseases in addition to lymphadenitis ${ }^{1}$. One of the species associated with lymphadenitis is Mycobacterium fortuitum, an intracellular parasite of fast growth resulting in a lesion that may evolve to a fistula and then to an ulcer. Lymphadenopathy occurs primarily in children and frequently attacks the lymph nodes in the neck, particularly those in the submandibular area. The systemic symptomatology is usually scarce, with no observation of inflammatory signs or pain.

\section{CASE REPORT}

A 9-year-old girl presented with palpable lymphadenomegaly on her left cervical region for approximately the previous 50 days, fever during the initial few days, and without any other systemic manifestations. From 3 days after the beginning of the clinical condition, the patient presented with typical symptomatology of varicella and spent 15 days resting during

Address to: Dr. Kleber Giovanni Luz. Rua Des. Túlio Bezerra de Melo 3631/ $\mathrm{Apt}^{\mathrm{O}}$ 1000, Bairro Candelária, 5964-580 Natal, RN, Brasil.

Phone: 5584 9982-4374

e-mail: klebergluz@gmail.com

Received 23 August 2013

Accepted 24 January 2014 which time the node continued to grow. During this period, a vesicle with purulent content was observed on her lower left gum. It was associated with a dental fracture that had occurred prior to the emergence of the node. Panoramic radiography showed no abnormalities. Owing to suspicion of a bacterial infection, amoxicillin was administered without clinical improvement.

As the clinical condition persisted, new laboratory exams and cervical ultrasound showed a left submandibular hypoechoic nodule. Owing to this finding, amoxicillin was ceased, and cephalexin treatment was initiated.

On day 51, lymphadenitis evolved with a fistula followed by a cutaneous suppurative ulceration. A biopsy indicated a left submandibular tumor with reactive lymphoid hyperplasia, the beginnings of epithelioid granuloma and necrotic areas, and negative results for alcohol acid resistant bacilli (AARB). Fungus growth in culture was not observed, and no microorganism growth was detected in the culture with an automated antibiogram of the fragment material of the submandibular region and cervical secretion. No alterations could be observed by thorax radiography. Non-reactant purified protein derivative (PPD) and fast anti-human immunodeficiency virus (HIV) tests were negative. Cephalexin was ceased, as it resulted in no clinical improvement.

A circular lesion was evidenced by physical examination. It measured $4.5 \mathrm{~cm}$ in diameter with well limited, prominent, hardened, and erythematosus edges; a granular base covered with purulent content; and a surrounding purple area. A biopsy was performed, and the material was sent for anatomopathology culture analysis that revealed only areas of necrotizing granulomatosis processes without evidence of infectious agents (Figure 1). Two palpable lymph nodes were identified at the mandible that were static, of hardened consistency, and painful to touch. The largest node measured $1.5 \mathrm{~cm}$ in diameter. No other lymph node chain was observed. 


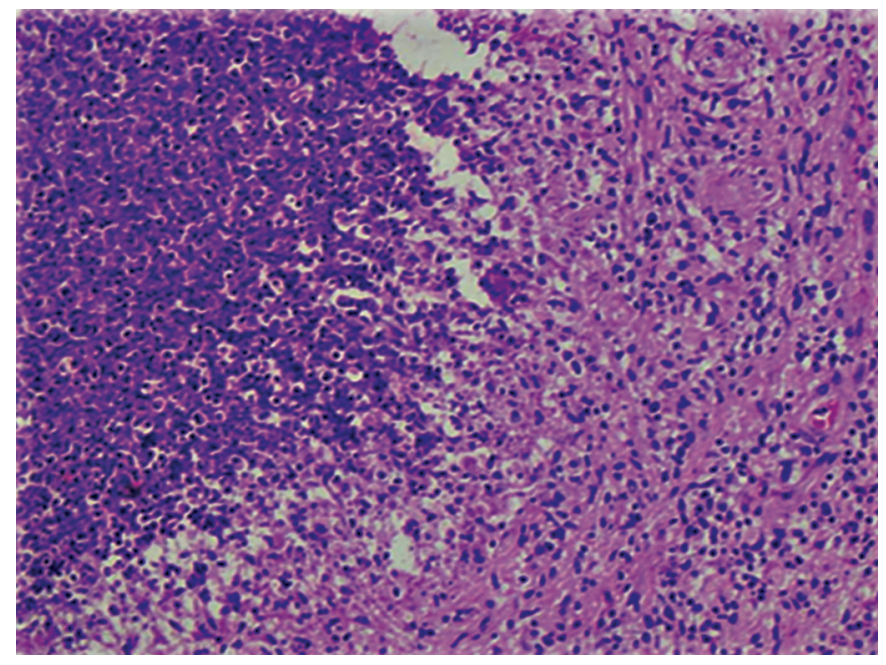

FIGURE 1 - Area of granulomatous necrotizing process in a lesion with well limited, prominent, hardened, and erythematosus edges.

Another sample of the cervical nodule was initially processed for culture to detect common germs, AARB, fungi, Actinomyces, and Nocardia; all of the cultures were negative. A part of the material was sown in culture media (lamb blood agar,
MacConkey agar, Sabouraud agar, Mycosel agar, LowensteinJensen medium, and Ogawa-Kudoh agar). The other part of the sample was cultivated in an automated microbial detection system (BacTALERT, BioMerieux, Inc., Durham, NC, USA). The sample in the flask turned positive after the fourth day of incubation and was identified as positive for AARB through the Ziehl-Neelsen method, suggesting growth in the LowensteinJensen and Ogawa-Kudoh media where the colonies presented a rough yellowish color ${ }^{3}$.

Growth was detected in the Lowenstein-Jensen medium with added paranitrobenzoic acid, niacin was produced, and the cord factor was absent. The findings indicated mycobacterium of rapid growth. The strains ATCC 6842, ATCC h37Ra, and ATCC 25177 were used as quality control culture media. To confirm the final minimum cell rate (MCR), a polymerase chain reaction (PCR) with DNA sequencing was performed; as a result, Mycobacterium fortuitum was identified ${ }^{4}$.

The sensitivity test results revealed sensitivity to imipenem with a minimum inhibitory concentration (MIC) of 4.0 and sulfametoxazol/trimetoprima with MICs of 9.5 and 0.5 , respectively. Treatment with imipenem + cilastatin sodium, which is associated with sulfametoxazol + trimetoprima, was initiated, resulting in a retraction of the lesion (Figure 2).
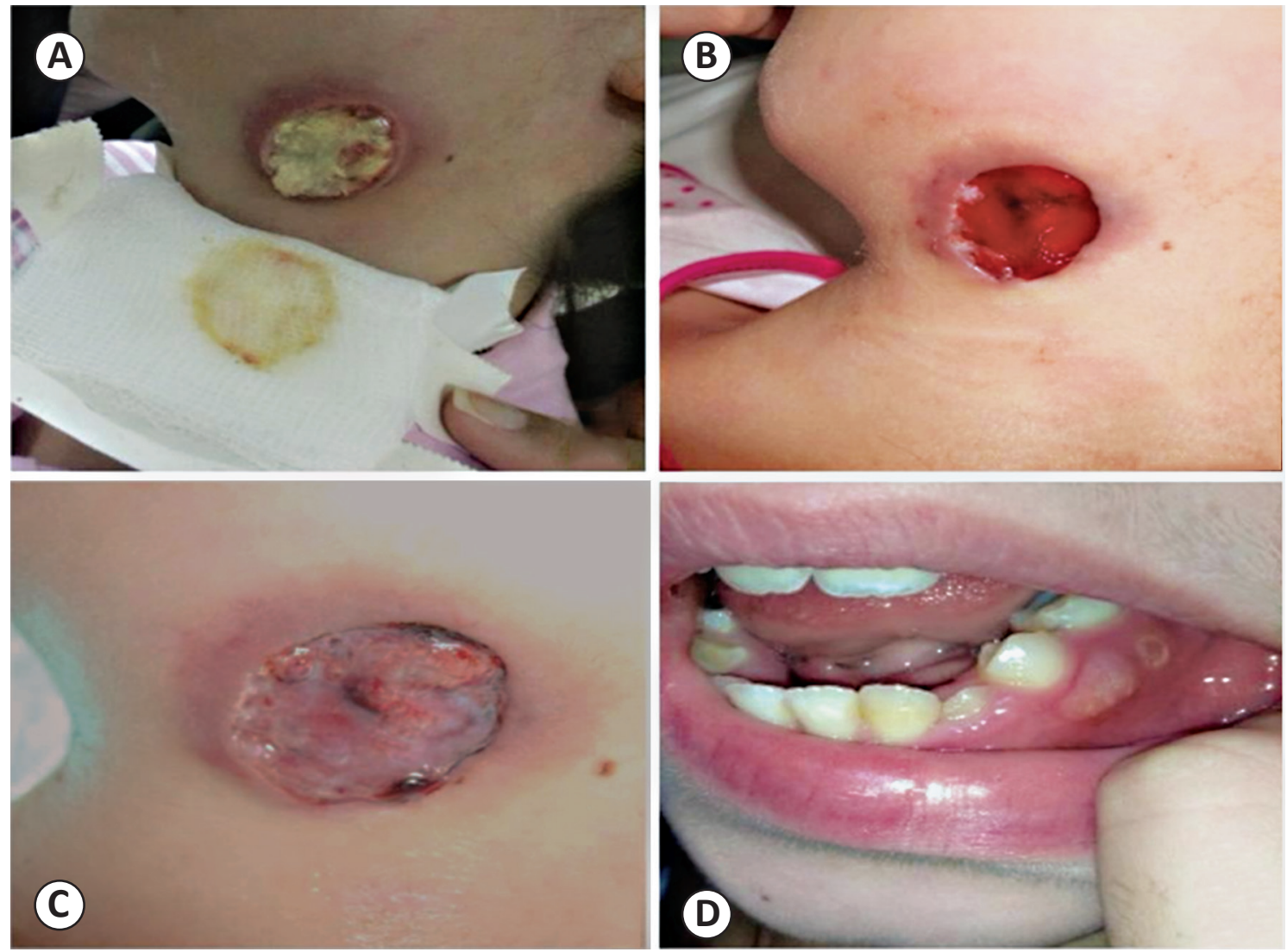

FIGURE 2 - A: Lesion after first biopsy. B: Lesion 2 days after second biopsy. C: Lesion 5 days after second biopsy. D: Vesicle with purulent content. 


\section{DISCUSSION}

Owing to the severity of the lesion, it was unclear how a patient who was otherwise healthy and without previous disease presented with such an extensive clinical manifestation. It is understood that a weakened immune system should be present for NTM to be able to colonize and cause disease in the host. In the present case, varicella zoster virus $(\mathrm{VZV})^{5}$ activity was observed, resulting in an impaired immune defense against the macrophages, the primary initial immune response agents against NTM.

VZV interferes with the process of phagocyte activation; it phosphorylates the signal transducer and activator of transcription (STAT) 3, a transcription activator that lowers the levels of STAT1 in the macrophage through negative feedback ${ }^{6}$. STAT1 is a transcription activator that acts directly in the nucleus by stimulating the production of interleukin (IL)-12 and oxidant agents (reactant species of oxygen [ERO] and nitric oxide $[\mathrm{NO}])^{7}$.

IL-12 recruits and activates T lymphocytes such as Th1, which produces interferon (IFN)-gamma'. IFN-gamma bonds to specific receptors (IFN gamma-R) in the macrophages, activating the kinase protein (JAKS) that phosphorylates and activates STAT1 ${ }^{7}$. However, VZV infection interferes with this signal transduction by antagonizing STAT3 with STAT 1 and decreasing the production of ERO and NO. Thus, oxidizing agents are overwhelmed by the production of antioxidant enzymes such as catalase, which is a resistance mechanism of mycobacterium. As a consequence, bacteria are phagocytosed but not destroyed, remaining in the interior of the macrophages, establishing their pathogenic potential, and resulting in clinical manifestations ${ }^{8}$.
Depending on the exposure, NTM should always be considered as possible causative agents for infection when presented with lymphadenitis; however, NTM has low potential for causing disease in healthy individuals. Related viral infections, such as varicella, were observed; such infections depress the immune system and promote alterations in the bacterial infectious process, thus affecting its severity.

\section{REFERENCES}

1. Cassidy PM, Hedberg K, Saulson A, McNelly E, Winthrop KL. Nontuberculous mycobacterial disease prevalence and risk factors: a changing epidemiology. Clin Infect Dis 2009; 49:124-129.

2. Falkinham OJ. Impact of human activities on the ecology of nontuberculous mycobacteria. Future Microbiol 2010; 5:951-960.

3. Idigoras $\mathrm{P}$, Beristain $\mathrm{X}$, Iturzaeta $\mathrm{A}$, Vicente $\mathrm{D}$, Pérez-Trallero E. Comparison of the automated nonradiometric bactec MGIT 960 system with Löwenstein-Jensen, Coletsos, and Middlebrook 7H11 Solid Media for recovery of mycobacteria. Eur J Clin Microbiol Infect Dis 2000; 19:350-354.

4. Falkinham OJ. Impact of human activities on the ecology of nontuberculous mycobacteria. Future Microbiol 2010; 5:951-960.

5. Belsey MA. Tuberculosis and varicella infections in children. Am J Dis Child 1967; 113:444-448.

6. Sen N, Che X, Rajamani J, Zerboni L, Sung P, Ptacek J, et al. Signal transducer and activator of transcription 3 (STAT3) and survivin induction by varicella-zoster virus promote replication and skin pathogenesis. PNAS 2011; 109:600-605.

7. Guide SV, Holland SM. Host susceptibility factors in mycobacterial infection: genetics and body morphotype. Infect Dis Clin North Am 2002;16:163-186.

8. Ottenhoff TH, Verreck FA, Hoeve MA, van de Vosse E. Control of human host immunity to mycobacteria. Tuberculosis (Edinb.) 2005; 85:53-64.

9. Stenger S, Modlin RL. T cell mediated immunity to Mycobacterium tuberculosis. Curr Opin Microbiol 1999; 2:89-93. 